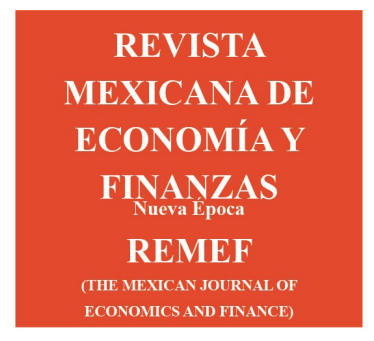

Revista Mexicana de Economía y Finanzas
Nueva Época
Volumen 15 Segundo Número Especial Aniversario
Agosto $2020 \quad$ pp. $503-521$

SNEA - Entrepreneurial research in emerging economies: recent advances Editor Invitado: Dr. Samuel Mongrut

\title{
Ecosistema global del emprendedor desde una perspectiva financiera y tecnológica
}

\author{
Martha del Pilar Rodríguez García ${ }^{1}$
}

Universidad Autónoma de Nuevo León, México

\section{Resumen}

Esta investigación tiene como objetivo realizar una comparativa del ecosistema emprendedor, financiero y tecnológico entre países desarrollados y emergentes. Se utiliza el método no paramétrico de Vecindades Cercanas con el fin de analizar diferencias entre países emergentes y desarrollados. Los hallazgos muestran que los ecosistemas globales permanecen sin cambios en el tiempo. Las recomendaciones son políticas públicas y privadas enfocadas a cambios cultures, inversión en infraestructura financiera y tecnológica. Existe escasa información en países como los africanos en donde es necesario que surjan los emprendimientos para la mejora del bienestar en su población. La metodología de Vecindades Cercanas nos permite la clasificación de países en grupos con similitudes relacionadas. El nivel de los ecosistemas financiero y tecnológico permanece casi estático durante el periodo de estudio entre las economías avanzadas y emergentes, lo que sitúa el nivel del emprendimiento a nivel internacional con pocos cambios.

Clasificación JEL: M13, M21

Palabras clave: ecosistema de emprendimiento, ecosistema financiero, ecosistema tecnológico, algoritmo de vecindades cercanas

\section{Global ecosystem of the entrepreneur from a financial and technological perspective}

\section{Abstract}

This research aims comparison of the entrepreneurial, financial, and technological ecosystem between developed and emerging countries. The non-parametric method of Nearest Neighbor is used to analyze differences between emerging and developed countries. The findings show that global ecosystems remain unchanged over time. The recommendations are public and private policies should be focus on cultural changes, investment in financial and technological infrastructure. There is a little information in countries such as Africa. The Nearest Neighbor methodology allows us to classify countries into groups with related similarities. The level of the financial and technological ecosystems remains almost static during the study period between developed and emerging economies, which places the level of entrepreneurship at the international level with little change.

\footnotetext{
${ }^{1}$ Facultad de Contaduría Pública y Administración, Pedro de Alba s/n, Ciudad Universitaria, San Nicolás de los Garza, C.P. 66455, (81) 134044 30, martha.rodriguezgc@uanl.edu.mx, ORCID: 0000-0001-8262-958X

${ }^{*}$ Sin fuente de financiamiento para el desarrollo de la investigación
} 


\section{Abstract}

JEL Classification: M13, M21

Keywords: Entrepreneur Ecosystem, Financial Ecosystem, Technological Ecosystem, Nearest Neighborhood algorithm

\section{Introducción}

Las oportunidades y retos que enfrentan los emprendedores cambian de una manera continua a nivel global. Schumpeter (1934) menciona que el emprendimiento es un proceso en el que los individuos aprovechan oportunidades para realizar innovaciones. Sin embargo, el fenómeno del emprendimiento empieza a tener un gran interés por parte de académicos y profesionales a finales de los noventas. En un estudio realizado por Malecki (2017) menciona que el ecosistema de emprendimiento se convierte en un tema dominante a partir del 2016. Es por ello, que la flexibilidad y la eficiencia del emprededurismo dependerá de las condiciones estructurales y sistémicas de la región en donde se encuentre el emprendedor.

En las economías avanzadas y emergentes el tópico del emprendimiento tiene un papel clave en el desarrollo económico (Ahlstrom y Bruton, 2008; Bruton et al., 2008). Audretsch (2003) menciona que en economías avanzadas las pequeñas y medianas empresas han sido los impulsores del empleo y de la innovación. Mientras que en economías emergentes el emprendimiento se origina por necesidad y su impacto es muy significativo para la creación de empleo, aunque no ha sido suficiente para la creación de empleo sostenible (Oladunjoye et al., 2011). Según un estudio de Wansleben (2013) se prevé que en el 2050 las economías de Brasil, China, India y Rusia (BRIC) serán más grandes que las economías conformadas por el G6, Francia, Alemania, Italia, Japón, Reino Unido y Estados Unidos.

El concepto de ecosistema se puede comprender mediante el estudio de los sistemas ecológicos, esto es, que se explica a través de la interacción de los organismos vivos con el entorno físico. El ecosistema puede mantenerse en equilibrio o estar abierto al exterior (McKenzie y Sud, 2009). La supervivencia de un ecosistema dependerá de la cooperación de los actores y los factores externos (O'Connor et al., 2018). Los resultados de la actividad emprendedora resultan de la creación de empresas innovadoras, empresas de alto crecimiento o como empleados emprendedores (Stam, 2014). En este trabajo empleamos la definición de ecosistema de emprendimiento que propone el Instituto de Desarrollo y Emprendimiento Global (en inglés, Global Entrepreneurship and Development Institute (GEDI) que define al ecosistema del emprendedor como: "la interacción dinámica, institucionalmente integrada entre actitudes empresariales, habilidades empresariales y aspiraciones empresariales de los individuos, lo que impulsa la asignación de recursos a través de la creación y operación de nuevas empresas." (Ács, et al., 2019).

Como señalan Bruton et al. (2008), existen muchos esfuerzos por parte de instituciones para fomentar el beneficio de un ecosistema del emprendedor fuentes como la Encuesta Global de Emprendimiento del Banco Mundial y el Monitor de Emprendimiento Global (Global Entrepreneur Monitor, GEM) son algunos ejemplos que ofrecen información pública. También existen trabajos de encuestas aplicadas por investigadores como en Avanzini (2011) y en Hofstede y Milosevic (2011) que ayudan a medir el ecosistema del emprendedor pero de una manera particular.

El Foro Económico Mundial señala que el acceso a los mercados de capitales son determinantes importantes para el crecimiento de las empresas. Asimismo, Harrington (2016) menciona que la medición del ecosistema del emprendedor está evolucionando en países, gobiernos y regiones, ya que reconocen que los nuevos emprendimientos impulsan buenos resultados económicos y sociales. Aunque se debería profundizar mejor en las causas fundamentales debido a que el acceso a los capitales depende de factores institucionales 
de los países (Acemoglu et al., 2005) y de los sesgos del emprendedor al tener un exceso de confianza pudiendo perjudicar al emprendimiento (Mongrut y Juárez, 2018).

El contexto tecnológico y financiero del emprendimiento es altamente heterogéneo como lo señala Autio et al. (2014), por lo que hoy más que nunca es importante analizar los efectos del crecimiento económico y de bienestar que genera un ecosistema del emprendedor. Además, existen diferentes tipos de medidas como las de conteo, actitud, ponderadas, macro, mixtas y finalmente las del ecosistema del emprendedor (Acs et al., 2014; Stam, 2018), siendo estas últimas las utilizadas en este trabajo.

El objetivo de esta investigación es realizar un análisis comparativo a nivel global del ecosistema del emprendedor desde una perspectiva financiera y tecnológica mediante la metodología de Vecindades Cercanas que nos permita la clasificación de países en grupos con similitudes relacionadas. Para ello, se utiliza una muestra de 43 países considerando seis regiones: Asia, Latinoamérica, África y Medio Oriente, Europa, Oceanía y Norte América. En este estudio se comparan dos periodos de estudio, esto es, 2006 y 2018. Los principales hallazgos de este trabajo de investigación muestran que en general existe un comportamiento estático en el ecosistema global del emprendedor y sobre todo en el grupo de niveles altos y bajos de emprendimiento, esto, ocasionado por diferencias culturales, de infraestructura y de regulaciones entre países. Asimismo, con relación al ecosistema financiero y tecnológico se encuentran grandes diferencias entre los dos años de estudio, 2006 y 2018, lo anterior debido a las grandes diferencias de apoyos financieros de capital y políticas de inclusión tecnológica en mercados desarrollados en contraste con los países emergentes.

Esta investigación contribuye al debate de si existe una movilidad en el tiempo entre grupos de países clasificados mediante atributos del ecosistema del emprendedor y si esto difiere cuando incluimos aspectos financieros y tecnológicos. De esta forma proveer de información y análisis a los generadores de políticas empresariales para que puedan visualizar las debilidades y fortalezas de los países bajo estudio con el fin de conocer las ventajas y desventajas de realizar un emprendimiento en una región o país determinado.

El trabajo se divide en cuatro secciones, en primer lugar, se presenta los antecedentes teóricos del ecosistema del emprendedor y la forma en que se mide este concepto, así como una revisión de los principales resultados de investigaciones entre países avanzados y emergentes en diversos continentes de Asia, África y Medio Oriente, Europa, Norteamérica, Latinoamérica y Oceanía. Posteriormente, se explica la metodología donde presentamos muestra y medición de nuestras variables, así como el método a utilizar. Finalmente, en las últimas dos secciones se analizan los resultados y conclusiones.

\section{Revisión de la literatura}

\subsection{Ecosistema de emprendimiento}

El estudio de los ecosistemas de emprendimiento tiene sus antecedentes teóricos en el estudio de la geografía económica, con sus precursores en la escuela californiana (Scott, 1986), la teoría de los clusters (Lawson, 1999), así como en la teoría ecléctica del emprendimiento (Verheul et al., 2002), entre otras. Las contribuciones en geografía económica principalmente se enfocan en el análisis de las interdependencias que las empresas pueden comercializar y las que no (Scott, 1986). Las principales contribuciones se relacionan con la división de trabajo y los costos de transacción que se incrementan con la distancia geográfica. Esta teoría se basa en las relaciones que se pueden comercializar (Lawson, 1999). La distancia geográfica es un elemento que aumenta o disminuye la interdependencia entre empresas. Por otra parte, en relación a las interdependencias no comercializables, Storper (1995) señala que el estudio de trayectorias tecnológicas, las regulaciones y la comunicación podrían mejorar las interdependencias entre empresas para el desarrollo del conocimiento.

En relación con la teoría de los grupos o clusters (también llamados "distrito industrial" o "distrito tec- 
nológico") autores como Porter (1998), Lawson (1999) y Tallman et al. (2004) mencionan que éstos permiten la creación del conocimiento mejorando la ventaja competitiva entre empresas mediante la disminución en costos de transacción y transportación. En este sentido, la pertenencia a un grupo o cluster les permite a sus miembros, un contexto en el que muchas empresas compiten simultáneamente, colaborar para ganar. Existen algunos casos de clústeres reconocidos como en la industria del cine "Hollywood", la industria del vino en California y el sector tecnológico en Silicon Valley y Boston. Algunas ventajas de estos grupos es el incremento de su comercio internacional y de sus inversiones, entre otras. Tanto la economía geográfica como la teoría de grupos o clusters se basan en las ventajas competitivas que proporcionan, ya sea la distancia geográfica o la cooperación entre empresas para el beneficio de menores costos, mayor especialización e innovación.

Existe una teoría adicional, que complementa a las anteriores, llamada Teoría Ecléctica del Emprendimiento (ETE) que explica como los aspectos macro de un país impulsan las actividades del emprendedor. Esta teoría es propuesta por Verheul et al. (2002) y proporciona un marco de referencia para conocer y analizar los factores que impulsan las actividades empresariales en un país. La ETE explica el nivel de emprendimiento mediante cuatro categorías de factores: la demanda (oportunidades económicas), la oferta (recursos y habilidades), calidad de gobierno y factores culturales. La ETE considera las cuatro categorías como variables exógenas a los empresarios, esto es, situación económica, desarrollo tecnológico y financiero y así como la globalización (Rousseau y Sylla, 2003; Verheul et al., 2002).

A partir de estas tres teorías profundizaremos en el término de "ecosistema del emprendedor" así como en sus mediciones para tratar de explicar los factores determinantes del mismo.

Existen muchos esfuerzos que han aportado académicos y profesionales (Reynolds, et al. 2005) para medir la evolución del emprendimiento global. Para ello, se realizó una revisión de las métricas del nivel del emprededurismo en un país o región y encontramos mediciones de conteo, de actitudes, institucionales, ponderadas y las que miden el ecosistema del emprendedor que serían una combinación de las anteriores.

Existen ejemplos tangibles como las mediciones basadas en el conteo que reportan actividades empresariales en una región determinada o país, pueden ser registros comerciales como por ejemplo los reportes del Banco Mundial, basados en encuestas o recuentos de tipos específicos de nuevas empresas. Por otra parte, existen esfuerzos para medir las actitudes individuales de los emprendedores, llamadas medidas actitudinales, que son usadas en las primeras etapas del emprendimiento y son una medición proxy de las prácticas culturales que enfrentan las personas en la gestión empresarial (Autio et al., 2014). En Henrekson (2005) menciona que son un reflejo de las instituciones informales a través de asignaciones de recursos sociales y el entorno regulatorio.

Desde el punto de vista de infraestructura existen las métricas que tienden a capturar instituciones más formales y condiciones estructurales tangibles (por ejemplo, nivel de educación de la población; calidad de las regulaciones e intervenciones de políticas de emprendimiento y la disponibilidad de recursos para el emprendimiento). Un ejemplo es el programa de "Doing Business" del Banco Mundial que se centra en obstáculos regulatorios. Otro es el Programa de Indicadores de Emprendimiento de la Organización para la Cooperación y el Desarrollo Económicos (OCDE) que mide aspectos de políticas y condiciones macroeconómicas (Ahmad y Hoffmann, 2008). La desventaja de estas mediciones es que los aspectos institucionales pudieran no medir la actividad empresarial.

Para capturar varias mediciones existen las medidas ponderadas, según Acs et al. (2014) combinan condiciones de contexto y resultados empresariales, proporcionando así un reflejo de la calidad de la dinámica empresarial en la economía. Algunos ejemplos de este tipo de medidas es el Índice Regional de Desarrollo y Emprendimiento (REDI).

Por último, se encuentran las medidas del ecosistema del emprendedor que representan la última evolución en la medición del emprendimiento (Stam, 2018). Estas medidas usan enfoques estructurados (Autio et al., 
2018) y además de reflejar la adopción generalizada de nuevas innovaciones de organización en la gestión de sus empresas. Asimismo, visualizan el contexto de operación de la empresa y los factores que le facilitan su gestión, esto es, su "ecosistema emprendedor" (Autio, et al., 2018).

Para un análisis más profundo sobre la visibilidad de actores y eventos clave que fomenten el ecosistema del emprendedor se puede recurrir al trabajo de Feld (2012) en donde señala que existen nueve atributos que facilitan el emprendimiento ${ }^{2}$. Por otra parte, en Isenberg (2010) menciona que no existe una fórmula en particular y propone nueve principios que enfatizan especialmente la sensibilidad a las condiciones locales, entre ellos, destaca la adaptación del ecosistema a las condiciones locales, incluir al sector privado, conocer el potencial, así como las reformas regulatorias necesarias entre otras. Por otra parte, en Foster et al. (2013) enumeran ocho pilares que conforman un ecosistema (exitoso), entre ellos, se destacan mercados externos e internos accesibles, talento de capital humano, acceso a financiación, sistemas de apoyo como servicios profesionales, regulaciones eficientes, educación que beneficie el emprendimiento, cultura de respeto al emprendimiento, cultura que promueva la tolerancia al riesgo y al fracaso. Cabe destacar este último como un pilar que no se menciona en Feld (2012) y en Isenberg (2010).

En Stam $(2014,2015)$ se propone un modelo basado en productos y resultados del ecosistema de emprendimiento. Para que esto se pueda presentar es necesario describir las condiciones sistémicas y las de infraestructura. Las condiciones de infraestructura o macro se miden por las instituciones formales e informales, cultura que permite o no la interacción humana en general, infraestructura física y la acción empresarial en especial la demanda. Por otra parte, las condiciones sistémicas (redes, liderazgo, finanzas, talento humano, nuevos conocimientos y servicios de apoyo) interactúan con cada uno y están restringidos y habilitados por las condiciones macro. Estas dos condiciones generan productos que serían medidos por emprendimientos innovadores, emprendimientos de alto crecimiento, y empleados emprendedores. Los resultados deben de estar relacionados con una creación de valor en la sociedad, medido por un incremento en la productividad, mayores ingresos, empleos y bienestar.

\subsection{Estudios comparativos}

En la revisión de la literatura se presentan hallazgos de diferentes autores en relación con los ecosistemas de emprendimiento en países de economías avanzadas y emergentes. En Voelker (2012) realiza un estudio global con una muestra de 69 economías avanzadas y en desarrollo para el periodo de 1998-2005. Sus principales hallazgos son que Japón y Estados Unidos tienen valores similares y no se agrupan con ningún otro país. Esto pudiera ser el resultado de altos niveles de investigación e innovación, aunque son diferentes en relación con su actividad empresarial. Por otra parte, encuentra que la permanencia en los grupos o clusters es estática en el tiempo, aunque puede tener cierta movilidad como en el caso de países como China, Rusia, la India e Indonesia. Asimismo, menciona que un grupo o cluster que tenga como fin la creación del conocimiento puede ser débil en generar nuevos negocios y viceversa. Además, menciona que la cultura juega un papel muy importante en el tipo de clúster en donde se encuentre el país.

En el tema de diferencias regionales o entre países, existen estudios que mencionan que el capital humano y la infraestructura, facilitan la creación del conocimiento y con ello impulsa la innovación y la creación de nuevas empresas que serán el resultado de la creación de valor a través de mayores niveles de empleo y crecimiento económico (Rosiello et al., 2011 y Stam (2015). En los países desarrollados se tienen adecuadas infraestructuras tanto de carácter financiero como social, además de buenas regulaciones y niveles de go-

\footnotetext{
${ }^{2}$ a) Liderazgo visible y accesible comprometido con la región, b) intermediarios representados por asesores respetados y bien integrados, c) redes para que los emprendedores estén conectados con inversores comprometidos, d) fortaleza del gobierno que incorpora políticas de desarrollo económico, fiscales e inversiones, e) talento humano, f) servicios legales y contables que sean accesibles, g) compromiso a través de eventos y ferias que permiten la competitividad, h) empresas ancla que fomenten el crecimiento de empresas pequeñas, i) capital semilla.
} 
bernanza en sus instituciones que favorecen la competitividad y el intercambio comercial. En contraste en las economías en desarrollo algunas, agrícolas o manufactureras, cuentan con sistemas financieros de poca inclusión, con infraestructura social y regulatoria básica que dificulta su competitividad (Voelker, 2012).

Las diferencias en educación y tecnología entre las economías avanzadas y las emergentes propician resultados diferentes en el crecimiento de nuevos emprendimientos (Yiu et al., 2007). En otros estudios se encuentran que dentro de las economías más avanzadas hay ecosistemas que potencian el desarrollo del emprendimiento (De Clercq et al., 2010). Aunque es importante destacar que, en países como Estados Unidos, las minorías enfrentan dificultades en la obtención de capital para sus empresas (Treichel y Scott, 2006) y provoca la dificultad de supervivencia de las mismas (Robb, 2002).

En Europa, Stam (2014) presenta un estudio aplicado al ecosistema de Países Bajos y sugiere que el gobierno debe proporcionar condiciones macro adecuadas en el área de educación, investigación, impuestos, protección al empleo, regulaciones del mercado, por mencionar algunas. Además, el estudio menciona que los países deben de realizar políticas macro que ayuden a estimular la movilidad laboral, al acceso de mercados de bienes y servicios públicos, así como realizar programas para estimular el liderazgo. Por último, remarca la generación del conocimiento entre institutos y universidades. Por otra parte, en Suecia una gran parte del presupuesto gubernamental se invierte en generar nuevos emprendimientos (Lundström et al., 2014). Además, es una de las principales economías en Europa con mayor nivel de digitalización (Gürdür et al., 2019).

Por último, es importante destacar que las diferencias culturales como la toma de riesgos y la confianza (Mihet, 2013) afectan los resultados económicos (Weber, 1965). En los países europeos son similares, ya que comparten un área geográfica común donde comercializan y comparten bienes y mano de obra intensiva, así como eventos históricos comunes como las guerras. Tabellini (2010) señala que la confianza es un valor cultural determinante en el desarrollo regional europeo. Por último, Harrintong (2016) señala que los cambios de política recomendados en Europa para favorecer el ecosistema del emprendimiento pudieran ser la reformulación de políticas a través de la difusión de las oportunidades de emprender. En relación con el ecosistema de emprendimiento en países emergentes, la situación económica muchas veces es débil (baja productividad, dificultad de fuentes de financiamiento, regulaciones no claras, etc.) el emprendimiento se inicia por necesidad (Oladunjoye et al., 2011) y a pesar de esto, origina crecimiento económico, pero no sostenible. En Voelker (2012) y Pušnik y Tajnikar, (2010) contemplan que el emprendimiento global como un proceso iterativo, esto es, que cambia y que en algún momento las economías emergentes pudieran pasar a comportarse como las avanzadas. En contraste, Raluca (2011) menciona que las diferencias en innovación e investigación dificulta este tipo de comportamientos. Existen casos de éxito en economías latinoamericanas como la chilena y la colombiana. En Chile en el 2008 se crean programas de investigación y desarrollo con beneficios fiscales generando gran aceptación por parte de empresas. En Colombia el Fondo Emprender se crea en el 2002 y apoyó a los nuevos emprendimientos con capital, además de ofrecer exenciones fiscales si se pagan los financiamientos. También, en ambos países, se han impulsado estrategias para mejorar la competitividad y la innovación (Herrington, 2017).

En economías asiáticas como en China la infraestructura física y la apertura del mercado son los mayores facilitadores para el emprendimiento. Existe una política de fomento del emprendimiento e innovación que se enfoca en reducir las barreras de entrada, fortalecimiento de los servicios públicos y alentar a estudiantes universitarios, científicos e ingenieros para comenzar nuevas empresas innovadoras (Herrington, 2017). Por otra parte, la tolerancia al riesgo es un valor que limita a este país. En Sudáfrica, se tienen problemas estructurales que no ayudan al crecimiento económico y del empleo. El emprendimiento es destructor en esta economía, es necesario promover la resiliencia en los emprendedores y eliminar los impedimentos culturales. Políticas de bienestar social impiden el desarrollo de las actividades de emprendimiento, así como también malas regulaciones y falta de fondos de capital privado y público (Geitlinger, 2016). En Harrintong (2016) se 
recomienda que en África Sahariana el gobierno apoye el nivel de educación a nivel bachillerato.

\section{Metodología}

\subsection{Muestra y medición}

Para la muestra se consideran 43 países tanto avanzados y emergentes según la clasificación más reciente del Banco Mundial (2020). El número de países está limitado a la muestra considerada en el GEM de 2006. En la Tabla 1 se presentan los países seleccionados por región geográfica (Asia, Latinoamérica, África y Medio Oriente Europa, Oceanía, Norte América), además se observa que el $55 \%$ de los países de la muestra se encuentran representados por economías avanzadas y el $45 \%$ por emergentes. Europa es la región con mayor número de países seleccionados, le sigue Latinoamérica y Asia.

Tabla 1. Muestra de los países por regiones y avance económico

\begin{tabular}{|c|c|c|c|}
\hline \multicolumn{2}{|c|}{ Economías avanzadas } & \multicolumn{2}{|c|}{ Economías emergentes } \\
\hline Región & País & Región & País \\
\hline Norte América & Estados Unidos & Asia & China \\
\hline Norte América & Canadá & Asia & Indonesia \\
\hline Europa & Alemania & Asia & Malasia \\
\hline Europa & Bélgica & Asia & Filipinas \\
\hline Europa & Croacia & Asia & Singapur \\
\hline Europa & Dinamarca & Asia & Tailandia \\
\hline Europa & Eslovenia & Asia & Rusia \\
\hline Europa & España & Asia & Turquía \\
\hline Europa & Finlandia & Asia & India \\
\hline Europa & Francia & Latinoamérica & Argentina \\
\hline Europa & Grecia & Latinoamérica & Brasil \\
\hline Europa & Hungría & Latinoamérica & Chile \\
\hline Europa & Irlanda & Latinoamérica & Colombia \\
\hline Europa & Islandia & Latinoamérica & Jamaica \\
\hline Europa & Italia & Latinoamérica & México \\
\hline Europa & Letonia & Latinoamérica & Perú \\
\hline Europa & Noruega & Latinoamérica & Uruguay \\
\hline Europa & Países Bajos & África & Sudáfrica \\
\hline Europa & Reino Unido & Medio Oriente & Emiratos Árabes Unidos \\
\hline Europa & República Checa & & \\
\hline Europa & Suecia & & \\
\hline Asia & Japón & & \\
\hline Oceanía & Australia & & \\
\hline
\end{tabular}

Podemos observar que dentro de la región asiática de la muestra sólo Japón es una economía clasificada como avanzada en contraste con la Europa en donde todos los países son considerados avanzados. Por último, cabe destacar que todos los países que se encuentran en las regiones de Latinoamérica, África y Oriente Medio están clasificados como emergentes. 
A partir de la metodología propuesta por el Instituto de Desarrollo y Emprendimiento Global (GEDI de sus siglas en inglés Global Entrepreneurship and Development Institute) se construye el Índice de Emprendimiento Global (GEI de sus siglas en inglés Global Entrepreneurship Index). En su versión más actualizada del GEI de 2019 emplea indicadores individuales e institucionales para medir el ecosistema del emprendedor (véase Tabla 2).

Tabla 2. Nueva estructura del Índice de Emprendimiento Global (GEI)

\begin{tabular}{|c|c|c|}
\hline Sub-índices & Pillars & Variables \\
\hline \multirow{10}{*}{ Actitudes } & \multirow{2}{*}{ Percepción de oportunidades } & Oportunidad de reconocimiento1 \\
\hline & & Libertad2 \\
\hline & \multirow{2}{*}{ Habilidades iniciales (Start-up skills) } & Percepción de oportunidad1 \\
\hline & & Habilidades educativas2 \\
\hline & \multirow{2}{*}{ Aceptación de riesgos } & Percepción del riesgo1 \\
\hline & & Riesgo país2 \\
\hline & \multirow{2}{*}{ Redes } & Conocimiento de emprendedores1 \\
\hline & & Urbanización e infraestuctura2 \\
\hline & \multirow{2}{*}{ Apoyo cultural } & Estado profesional1 \\
\hline & & Corrupción2 \\
\hline \multirow{8}{*}{ Habilidades } & \multirow{2}{*}{ Oportunidades iniciales } & Oportunidad de motivación1 \\
\hline & & Gobernanza2 \\
\hline & \multirow{2}{*}{ Absorción tecnológica } & Nivel tecnológico1 \\
\hline & & Absorción tecnológica2 \\
\hline & \multirow{2}{*}{ Capital humano } & Nivel educativo1 \\
\hline & & Mercado laboral2 \\
\hline & \multirow{2}{*}{ Competencia } & Competidores1 \\
\hline & & Competencia2 \\
\hline \multirow{10}{*}{ Aspiraciones } & \multirow{2}{*}{ Innovación de productos } & Nuevo producto1 \\
\hline & & Transferencia tecnológica2 \\
\hline & \multirow{2}{*}{ Innovación en procesos } & Nueva tecnología1 \\
\hline & & Investigación2 \\
\hline & \multirow{2}{*}{ Alto crecimiento } & Gacela (empresa de alto crecimiento) 1 \\
\hline & & Financiación y estrategia2 \\
\hline & \multirow{2}{*}{ Internacionalización } & Exportaciones1 \\
\hline & & Complejidad2 \\
\hline & \multirow{2}{*}{ Capital de riesgo } & Inversión informal1 \\
\hline & & Fondos de mercados de capital2 \\
\hline
\end{tabular}

1 Variables individuales

2 Variables institucionales

Fuente: Consultado en Ács, et al., (2019)

Los indicadores individuales son proporcionados por el GEM que aplica una encuesta llamada "Adult Population Survey" que captura aspectos del emprendedor y de la empresa considerando 43 países que tienen información desde el 2006 (Ács, et al., 2019). En relación con los indicadores institucionales o macro provienen principalmente del Global Competitiveness Index (GCI) del World Economic Forum (WEF) (13 de 20 indicadores institucionales), el resto de diversas fuentes secundarias ${ }^{3}$.

\footnotetext{
${ }^{3}$ Utiliza fuentes secundarias para los indicadores institucionales como el Índice de Percepción de la Corrupción, de la UNESCO
} 
A partir de información de carácter individual e institucional, el GEI construye cinco niveles de información como: indicadores, variables, pilares, subíndices y el super-índice. Estos indicadores se obtuvieron del sitio Instituto de Desarrollo y Emprendimiento Global que proporciona el GEI ${ }^{4}$. Los indicadores son el nivel de medición más simple o básico que puede ser del tipo individual o institucional. Estos indicadores pueden provenir de encuestas o de fuentes secundarias. Por otra parte, en la Tabla 2 se presentan las variables que surgen a partir de un conjunto de indicadores. Asimismo, el GEI contempla 14 pilares que se definen para una mejor comprensión (véase Tabla 2) y menciona que éstos son los más importantes constituyentes del súper-índice GEI (Ács, et al., 2019).

El segundo de ellos contempla las habilidades empresariales del emprendedor para determinar si las nuevas empresas tienen potencial de crecimiento, el potencial de intensidad tecnológica de la empresa, el nivel de educación del emprendedor, el nivel inicial de competencias y las capacidades digitales. En relación con los factores institucionales se contemplan aspectos tributarios y la eficiencia de la operación del gobierno, la capacidad de absorción de tecnología, la libertad y la posibilidad de capacitación en el mercado laboral. Además, el dominio de grupos empresariales y la regulación anti-monopolio.

Por último, el sub-índice de aspiración empresarial, se contemplan factores variables individuales la generación de nuevos productos y tecnologías por parte de los emprendedores, empresas de alto crecimiento llamadas gacelas, exportaciones inversiones informales. Por otra parte, se incluyen factores institucionales como la transferencia de tecnología, el potencial de investigación aplicada de la ciencia, la disponibilidad de capital de riesgo y la sofisticación de la estrategia, la internacionalización y la disponibilidad de financiamiento.

La información estadística de los pilares y de los sub-índices fueron consultados de la página del GEI ${ }^{5}$ y a partir de esta información se realizaron las clasificaciones del ecosistema del emprendedor como más adelante se explica.

\subsection{Método de vecindades cercanas}

En esta investigación se emplea el método de agrupamiento o clasificación llamado Vecindades Cercanas (VC) (Nearest neighborhood, en inglés). Este método es no paramétrico y utiliza la correlación espacial entre los puntos de un espacio de fase. El método de VC ha sido empleado en tareas de clasificación (Covery y Hart,1967; Dasarathy, 1991) y ampliamente utilizado en el reconocimiento de patrones y de la automatización del aprendizaje (Xu, et al., 2013 y Zang, et al., 2017). Existen diversas aplicaciones en el área de ingeniería (Jain y Dubes, 1998), en el área climatológica (Lorenz,1969), en la medicina para la predicción de enfermedades cardíacas a partir de los síntomas, así como también en la predicción del tipo de cambio (Fernández-Rodríguez et al., 2004).

Aunque existen diferentes algoritmos para clasificar objetos en grupos, el algoritmo de VC es considerado como de simple implementación, alta efectividad e intuitivo, por lo que se considera como uno de los diez mejores algoritmos en minería de datos (Wu et al, 2004). La elección de este tipo de algoritmo se determinó, ya que el propósito de esta investigación es encontrar grupos de países a través de las similitudes de un conjunto de variables de tal forma que la distancia entre los países de un mismo grupo de vecinos (k) sea mínima (Sur et al., 2013).

En esta investigación utilizamos el método de VC para reconocer patrones, en primera instancia, a partir

(educación terciaria), de las Naciones Unidas (urbanización), de la fundación Heritage y del Banco Mundial (libertad económica, derechos de propiedad, libertad laboral, buena gobernanza), del Observatorio de Complejidad Económica (complejidad económica), de la OCDE (riesgo país) y el Índice de atractividad del País y Capital Riesgo y Privado (fondos del mercado de capitales).

${ }^{4}$ https://thegedi.org/datasets/ Recuperado en: 2 de marzo del 2020.

${ }^{5}$ Sitio consultado para la base de datos: https://thegedi.org/datasets/ 
de tres sub-índices del ecosistema del emprendedor: actitudes, habilidades y aspiraciones. Posteriormente, para el análisis de los pilares financieros y tecnológicos, lo que nos ayudará a encontrar patrones similares entre países y el cambio que han tenido en el tiempo entre dos años de estudio 2006 y 2018.

El procedimiento a seguir se puede encontrar en Stone (1977), Sugihara y May (1990), y Finkenstadt y Kuhbier (1995). El algoritmo se describe en base a Finkenstadt y Kuhbier (1995) y Arroyo y Maté (2009) de la siguiente forma pero considerando series de corte transversal:

a) La información de una serie de corte transversal i, $\left\{X_{i}\right\}$ coni $=1, \ldots, n$, es transformada en series de vectores de d-dimensional, donde d representa el número total de variables, esto es, la serie resultante de vectores se denota por $X_{i}^{d}$ cond $=1, \ldots, \mathrm{p}$, donde $X_{i}^{d}=\left(X_{i}^{1}, X_{i}^{2}, \ldots, X_{i}^{p}\right)$ y representa un punto en el espacio d-dimensional.

b) La distancia entre el vector focal $X_{f}^{d}=\left(X_{f}^{1}, X_{f}^{2}, \ldots, X_{f}^{p}\right)$ y cada vector en la serie de corte transversal $\left\{X_{i}^{d}\right\}$ es computada, y el vector k más cercano a $X_{f}^{d}$ es seleccionado. La distancia utilizada en este estudio es la suma de todas las dimensiones de la diferencia absoluta entre los valores de los casos (ya sea sub-índices o pilares), también llamada distancia de Manhattan.

De esta forma el resultado de las clasificaciones por nivel (bajo, medio, y alto) se obtienen a partir de tres criterios (véase Tabla 2): a) clasificación del ecosistema del emprendedor mediante los tres sub-índices del GEI (actitudes, habilidades y aspiraciones), 2) clasificación del ecosistema financiero (aceptación de riesgos, alto crecimiento y capital de riesgo) y por último, b) clasificación del ecosistema tecnológico (absorción tecnológica, innovación de productos e innovación de servicios).

Para cada criterio se siguen las siguientes etapas:

1. Selecciona el país que tenga el más alto GEI como punto focal.

2. Buscar los 12 vecinos (que representa la tercera parte de la muestra) más próximos utilizando los tres sub-índices que componen el GEI, utilizando la distancia de Manhattan. ${ }^{6}$

3. Realizar la gráfica en el espacio de fases y determinar los doce vecinos más próximos al país focal.

4. Se vuelven a realzar los pasos del punto 1 al 3 pero utilizando el país con el GEI más bajo.

5. De los doce países de la muestra restantes se construye el grupo del nivel medio, para ello, se considera como país focal el que tenga la distancia más cercana entre su GEI y el promedio de los doce países que quedaron de tal forma que cubra dentro de sus vecinos la mayoría de los países faltantes.

Para el caso de las clasificaciones del ecosistema financiero y tecnológico se utilizaron como países focales los del valor promedio más alto o bajo, según el nivel que corresponda, de los tres pilares seleccionados respectivamente.

\section{Resultado}

\subsection{Ecosistema global del emprendedor}

A continuación, se presentan los tres grupos del ecosistema del emprendimiento generados por el método de clasificación de VC y considerando los tres sub-índices del GEI: nivel alto, medio y bajo. En la Gráfica

\footnotetext{
${ }^{6}$ También conocida como métrica de bloques de ciudad, distancia entre dos casos es la suma, en todas las dimensiones, de las diferencias absolutas entre los valores de esos casos.
} 
1 se presentan los tres niveles o grupos del 2006 y en la Gráfica 2 los niveles generados en el 2018. En el 2006 el nivel alto corresponde a los 12 países más parecidos al país con mayor GEI, en este caso, el país líder estuvo representado por Canadá. Cabe destacar que los países clasificados en el nivel 1 son de la región de Norte América (Estados Unidos), de Europa, países escandinavos (Suecia, Noruega, Islandia. Finlandia, Dinamarca) de la Unión Europea (Irlanda, Países Bajos, Bélgica, Alemania y Francia), así como Gran Bretaña y de Oceanía, Australia.

En el 2018 la posición de líder es de Estados Unidos (véase Gráfica 2) representando el nivel alto de ecosistema del emprendedor los 13 vecinos más próximos se mantienen durante 12 años de manera estática. Esto es consistente estudios que mencionan que el capital humano y la infraestructura (Rosiello et al., 2011; Stam, 2015) favorecen la creación de nuevas empresas en las economías más avanzadas (De Clercq et al., 2010). Asimismo, los valores de algunos países europeos y la distancia geográfica favorecen el intercambio de productos y de mano de obra; además de que comparten memoria histórica como guerras (Tabellini, 2010). Este comportamiento estático de los países es consistente con Raluca (2011) que menciona que los conocimientos generados en ciertos países ayudan a que este tipo de países lleven la delantera en el desarrollo económico.

El grupo correspondiente al nivel medio en el ecosistema del emprendedor en el 2006 tiene como líder a un país europeo, Letonia y a países de Asia (Malasia, Singapur, Japón), Latinoamérica (Chile y Uruguay), Europa (España, Italia, Grecia, República Checa), así como países árabes, Emiratos Árabes Unidos y de África, Sudáfrica. El nivel medio de emprendimiento de este grupo en el 2018 fue el que más experimentó cambios en sus miembros ya que se conservó en $64 \%$ la totalidad de miembros con respecto al 2006 y el $36 \%$ se movió a niveles bajos como Malasia, India, Uruguay y Sudáfrica. Esto es consistente con lo que señalan Oladunjoye et al. (2011) que menciona que en los países emergentes la baja productividad y las regulaciones no claras no ayudan a mejorar el nivel de emprendimiento de este grupo de países en este caso el $36 \%$ de los países de este nivel bajaron de nivel medio a bajo.
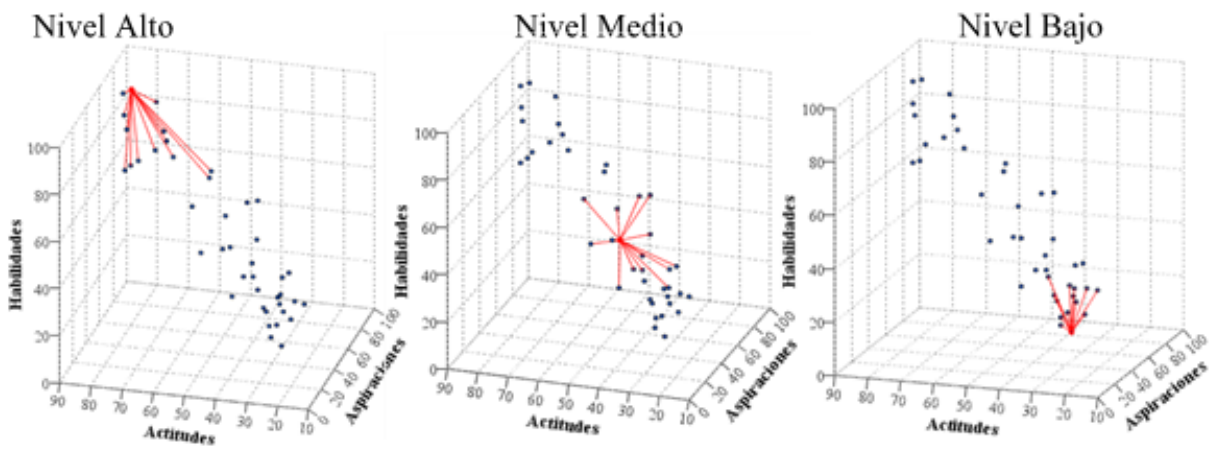

Gráfica 1. Ecosistema del emprendedor: 2006. Fuente: GEI, elaboración propia con SPSS21.
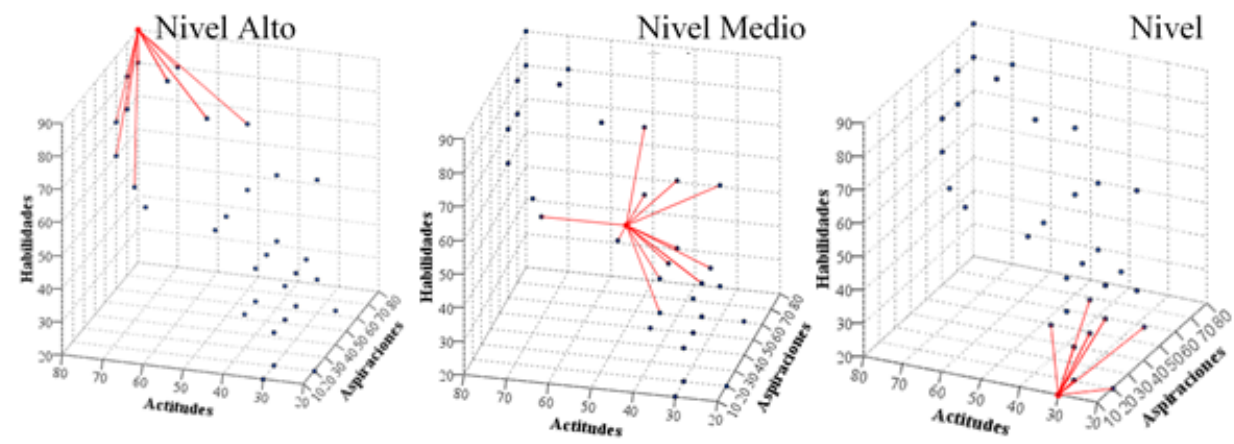

Gráfica 2. Ecosistema del emprendedor: 2018. Fuente: GEI, elaboración propia con SPSS21. 
El nivel bajo del ecosistema del emprendimiento (véase Gráfica 1) tiene como líder durante el 2006 a Filipinas. Los integrantes de este nivel bajo de emprendimiento se encuentran integrados por países latinoamericanos (Jamaica, Brasil, Perú, Argentina, Colombia, México), Asia y Europa Oriental (Indonesia, China, Tailandia, Turquía, Rusia) y de la Unión Europea (Hungría y Croacia). En la Gráfica 2, durante el año 2018, el líder de nivel bajo de emprendimiento es Jamaica, y el cambio de los integrantes del 2006 al 2018 se mantiene en un $71 \%$ de sus miembros siendo sólo cuatro países los que cambian de bajo a medio como China, Colombia Turquía y Hungría.

Voelker (2012) y Pušnik y Tajnikar, (2010) contemplan al emprendimiento global como un proceso iterativo, esto es, que cambia y que en algún momento las economías emergentes pudieran pasar a comportarse como las avanzadas, en este caso casos como el de China y Colombia son ejemplos claros de esta situación. Herrington (2017) señala que la economía de China ha mejorado su infraestructura y ha eliminado las barreras, lo cual ha generado un mejor ecosistema empresarial. Por otra parte, una economía que ha mejorado su ecosistema del emprendedor es Colombia en donde el gobierno ha instrumentado estrategias de fomento a la innovación como fondos a las pequeñas y medianas empresas, entre otros.

\subsection{Ecosistema financiero del emprendedor}

En relación con el ecosistema financiero para el emprendimiento en la Tabla 3 se presentan los tres niveles obtenidos en el 2006 y en la Tabla 4 los niveles en el 2018. Estados Unidos permanece como líder y país focal y en las tablas se observan que las distancias promedias en este nivel aumentan de 41.57 a 62.76 . Además, países como Bélgica, Suecia y Emiratos Árabes Unidos, salen del nivel alto y entran Chile, China y Francia (véase Tabla 4). Estos hallazgos son consistentes con Voelker (2012) que menciona que los países desarrollados tienen fortalezas en la infraestructura financiera por lo que se mantienen. Por otra parte, Herrintong (2017) menciona importantes políticas financieras en países Chile y China, creando fondos en inversiones de capital en empresas estratégicas en ambos países.

Tabla 3. Vecinos más próximos (k=14): Ecosistema financiero para el emprendimiento 2006.

\begin{tabular}{|c|c|c|c|c|c|}
\hline \multicolumn{2}{|c|}{ Nivel Alto } & \multicolumn{2}{c|}{ Nivel Medio } & \multicolumn{2}{c|}{ Nivel Bajo } \\
\hline País & Distancias & País & Distancias & País & Distancias \\
\hline Estados Unidos1 & 0.00 & China1, 2 & 0.00 & Filipinas1 & 0.00 \\
\hline Canadá & 21.20 & Italia & 32.40 & Jamaica & 21.20 \\
\hline Países Bajos & 25.30 & España & 40.60 & Brasil & 33.60 \\
\hline Singapur & 29.10 & Finlandia & 41.50 & Uruguay & 43.40 \\
\hline Reino Unido & 33.30 & República Checa & 45.20 & Rusia & 43.50 \\
\hline Dinamarca & 35.20 & Hungría & 48.50 & Indonesia & 52.20 \\
\hline Irlanda & 36.60 & Eslovenia & 51.10 & Perú & 53.10 \\
\hline Islandia & 40.00 & India2 & 56.20 & Tailandia & 57.30 \\
\hline Bélgica2 & 43.70 & Alemania & 61.70 & Malasia & 62.30 \\
\hline Suecia2 & 45.20 & Colombia & 64.90 & México & 71.50 \\
\hline Emiratos Árabes & 54.10 & Chile2 & 67.00 & Turquía2 & 73.40 \\
\hline Unidos2 & & Grecia & 73.20 & Argentina & 75.00 \\
\hline Noruega & 55.40 & Letonia & 74.30 & Croacia & 81.40 \\
\hline Japón & 59.50 & Francia2 & 79.20 & Sudáfrica & 82.70 \\
\hline Australia & 61.80 & Promedio & $\mathbf{5 6 . 6 0}$ & Promedio & $\mathbf{5 7 . 7 4}$ \\
\hline Promedio & $\mathbf{4 1 . 5 7}$ & Comed & & \\
\hline País & & & & \\
\hline
\end{tabular}

Notas: 1 País utilizado como focal según nivel de emprendimiento.; 2 País con cambio de nivel 2006 vs 2018.

Fuente: GEI elaboración propia con SPSS21. 
Tabla 4. Vecinos más próximos (k=14): Ecosistema financiero para el emprendimiento 2018.

\begin{tabular}{|c|c|c|c|c|c|}
\hline \multicolumn{2}{|c|}{ Nivel Alto } & \multicolumn{2}{|c|}{ Nivel Medio } & \multicolumn{2}{|c|}{ Nivel Bajo } \\
\hline País & Distancias & País & Distancias & País & Distancias \\
\hline Estados Unidos1 & 0.00 & Bélgica1, 2 & 0.00 & Jamaica1 & 0.00 \\
\hline Singapur & 23.90 & Suecia2 & 24.50 & Brasil & 24.60 \\
\hline Reino Unido & 47.00 & Alemania & 34.20 & Indonesia & 32.10 \\
\hline Noruega & 59.00 & República Checa & 40.80 & Filipinas & 45.10 \\
\hline Chile2 & 59.10 & Finlandia & 47.70 & Argentina & 53.10 \\
\hline Irlanda & 59.20 & Italia & 50.70 & Rusia & 53.60 \\
\hline Islandia & 64.10 & España & 52.40 & India2 & 54.70 \\
\hline Japón & 64.80 & Hungría & 66.60 & Tailandia & 56.90 \\
\hline Países Bajos & 65.70 & Eslovenia & 70.20 & México & 58.20 \\
\hline Francia2 & 68.20 & Letonia & 73.40 & Malasia & 63.50 \\
\hline Australia & 71.80 & Turquía2 & 78.50 & Uruguay & 72.70 \\
\hline Dinamarca & 75.10 & Colombia & 81.40 & Croacia & 73.80 \\
\hline China2 & 75.40 & $\begin{array}{c}\text { Emiratos Árabes } \\
\text { Unidos2 }\end{array}$ & 93.70 & Sudáfrica & 84.30 \\
\hline Canadá & 82.60 & Grecia & 95.40 & Perú & 87.70 \\
\hline Promedio & 62.76 & Promedio & 62.27 & Promedio & 58.48 \\
\hline
\end{tabular}

Notas: 1 País utilizado como focal según nivel de emprendimiento; 2 País con cambio de nivel 2006 vs 2018.

Fuente: GEI elaboración propia con SPSS21.

En el grupo de nivel medio se presentan los mayores cambios entre sus miembros comparando 2006 y 2018. En la Tabla 4 se observa que en el 2006 el líder es China, con sus respectivos países, entre ellos España y Chile. Durante el 2018 se puede observar que 36\% (4 de 14) de este nivel medio, sólo Turquía pasó de nivel bajo a medio, mientras que otros países como Suecia, Bélgica y Emiratos Árabes Unidos bajaron del nivel alto a medio. Lundström et al. (2014) mencionan que países como Suecia invierten grandes capitales por parte del gobierno, pero en algunas ocasiones en economías avanzadas como Finlandia, Países Bajos y Francia orientadas a la investigación pudieran no reflejar nuevos emprendimientos (Voelker, 2012).

Durante este año el nivel bajo de ecosistema financiero está conformado por países latinoamericanos, de Europa y Asia Oriental y de la Unión Europea. En la Tabla 4 se observa que hay cambio de líder y que el ecosistema financiero no se sustituye, ya que sólo Turquía sale de este grupo y se incorpora la India. El tipo de emprendimiento en estos países se caracteriza de subsistencia y no se apoya al emprendimiento, aquí las diferencias en educación entre las economías avanzadas y las emergentes propician resultados diferentes (Yiu et al., 2007) y desafortunadamente se mantienen en el tiempo.

\subsection{Ecosistema tecnológico para el emprendimiento}

En relación con el ecosistema tecnológico del emprendedor en la Tabla 5 y Tabla 6 se presentan los tres niveles obtenidos durante 2006 y 2018, respectivamente. Japón es líder en el 2006 y Bélgica en el 2018. En las tablas se observan que las distancias promedias en este ecosistema tecnológico disminuyen de 49.52 a 36.06, caso contrario ocurre en el ecosistema financiero. Además, países como Noruega e Islandia salen del nivel alto y países como Reino Unido y Países Bajos entran (véase Tabla 6). En el grupo de nivel medio, el líder es España en el 2006 e India en el 2018. Durante el 2018 se puede observar en la Tabla 6 que solo tres países cambiaron a este nivel medio, como Italia. Estos resultados refuerzan la idea que países avanzados como Japón y Bélgica el emprendimiento productivo es predominante en los países más cercanos a la frontera tecnológica (Ács, et al., 2019). Además ,Voelker (2012) menciona que países como, Bélgica se caracterizan por sus centros de investigación que pudiera estar fortaleciendo la parte de un ecosistema del emprendedor 
tecnológicamente más fuerte. En el mismo estudio encuentra que México, Argentina, Brasil y Sudáfrica no tengan fortalezas empresariales, en la misma línea de los hallazgos de la parte tecnológica.

Durante este año el nivel bajo de ecosistema tecnológico está constituido por países latinos, algunos europeos y asiáticos. En la Tabla 5 se observa que Filipinas es su líder y cambia en el 2018 por Jamaica (véase Tabla 6). Es importante destacar que solamente un país de los catorce cambia de grupo, lo que suponen que en la parte tecnológica este nivel bajo se mantiene estático. En Autio et. al (2014) menciona que el contexto tecnológico altamente heterogéneo, y las diferencias entre los países se mantienen en el tiempo (Yiu et al., 2007) y no aportando a su competitiva (Tallman et al., 2004).

Tabla 5. Vecinos más próximos (k=14): Ecosistema tecnológico del emprendedor 2006.

\begin{tabular}{|c|c|c|c|c|c|}
\hline \multicolumn{2}{|c|}{ Nivel Alto } & \multicolumn{2}{|c|}{ Nivel Medio } & \multicolumn{2}{|c|}{ Nivel Bajo } \\
\hline País & Distancias & País & Distancias & País & Distancias \\
\hline Japón1 & 0.00 & España1 & 0.00 & Filipinas1 & 0.00 \\
\hline Finlandia & 24.30 & Uruguay & 13.60 & Jamaica & 12.80 \\
\hline Estados Unidos & 27.90 & Argentina & 27.80 & Brasil & 51.00 \\
\hline Francia & 30.90 & Sudáfrica & 32.60 & Hungría & 59.20 \\
\hline Suecia & 35.30 & Malasia2 & 35.40 & Colombia & 60.70 \\
\hline Dinamarca & 37.80 & India & 45.70 & Perú & 62.20 \\
\hline República Checa & 46.80 & Turquía & 46.10 & Indonesia & 64.00 \\
\hline Canadá & 47.10 & China & 47.30 & Croacia & 65.10 \\
\hline Singapur & 47.80 & Reino Unido2 & 56.20 & Letonia & 66.60 \\
\hline Alemania & 55.20 & Chile & 58.90 & México & 67.50 \\
\hline Bélgica & 66.90 & Eslovenia & 65.60 & Grecia & 69.40 \\
\hline Islandia2 & 69.00 & Países Bajos2 & 66.10 & Rusia & 73.80 \\
\hline Noruega2 & 74.10 & Australia & 78.00 & Italia2 & 79.10 \\
\hline Irlanda & 80.60 & $\begin{array}{c}\text { Emiratos Árabes } \\
\text { Unidos }\end{array}$ & 94.80 & Tailandia & 79.80 \\
\hline Promedio & 49.52 & Promedio & 51.39 & Promedio & 62.40 \\
\hline
\end{tabular}

Notas: 1 País utilizado como focal según nivel de emprendimiento; 2 País con cambio de nivel 2006 vs 2018.

Fuente: GEI elaboración propia con SPSS21.

Tabla 6. Vecinos más próximos (k=14): Ecosistema tecnológico del emprendedor 2018.

\begin{tabular}{|c|c|c|c|c|c|}
\hline \multicolumn{2}{|c|}{ Nivel Alto } & \multicolumn{2}{c|}{ Nivel Medio } & \multicolumn{2}{c|}{ Nivel Bajo } \\
\hline País & Distancias & País & Distancias & País & Distancias \\
\hline Bélgica & 0.00 & India & 0.00 & Jamaica & 0.000 \\
\hline Francia & 14.60 & $\begin{array}{c}\text { Emiratos Árabes } \\
\text { Unidos }\end{array}$ & 23.10 & Brasil & 13.700 \\
\hline Japón & 21.20 & China & 43.20 & Perú & 33.300 \\
\hline Estados Unidos & 27.90 & Sudáfrica & 49.70 & Rusia & 38.400 \\
\hline Singapur & 30.00 & Turquía & 54.10 & México & 45.200 \\
\hline
\end{tabular}




\begin{tabular}{|c|c|c|c|c|c|}
\hline Irlanda & 31.10 & Filipinas & 55.00 & Argentina & 49.700 \\
\hline Canadá & 35.60 & Chile & 71.20 & Indonesia & 63.600 \\
\hline Alemania & 38.00 & Italia2 & 87.50 & Letonia & 67.500 \\
\hline Suecia & 40.50 & Uruguay & 100.00 & Hungría & 71.100 \\
\hline Reino Unido2 & 42.10 & España & 116.50 & Tailandia & 73.100 \\
\hline República Checa & 45.30 & Australia & 139.50 & Grecia & 76.200 \\
\hline Dinamarca & 46.30 & Eslovenia & 149.10 & Croacia & 83.000 \\
\hline Países Bajos2 & 47.20 & Noruega2 & 151.10 & Colombia & 85.500 \\
\hline Finlandia & 49.00 & Islandia2 & 165.70 & Malasia2 & 105.600 \\
\hline Promedio & $\mathbf{3 6 . 0 6}$ & Promedio & $\mathbf{9 2 . 7 5}$ & Promedio & $\mathbf{6 1 . 9 9 2 3}$ \\
\hline
\end{tabular}

Notas: 1 País utilizado como focal según nivel de emprendimiento; 2 País con cambio de nivel 2006 vs 2018.

Fuente: GEI elaboración propia con SPSS21.

\section{Conclusiones}

Los hallazgos de esta investigación proporcionan una serie de conclusiones y tendencias. En primer lugar, el efecto del área geográfica y la necesidad económica de algunos emprendedores pueden ser determinantes de su ecosistema (Rousseau y Sylla, 2003; Verheul et al., 2002; Bruton et al., 2008). En este sentido, construimos tres ecosistemas del emprendedor: global, financiero y tecnológico. Encontrando diferentes comportamientos en unos más estáticos (cerrados) y en otros más dinámicos (abiertos).

Se presenta un comportamiento estático en el ecosistema global del emprendedor en el nivel alto y bajo, esto puede ser ocasionado por niveles altos de investigación e innovación en los países desarrollados y bajos niveles en países emergentes, así como por diferencias culturales (Voelker, 2012). Además, las diferencias en infraestructura, regulaciones y niveles de gobernanza (Stam, 2015) ocasionan que el ecosistema del emprendedor tenga poca movilidad de sus miembros en el tiempo. Solamente, economías como China, Colombia Turquía y Hungría han logrado subir al nivel medio de emprendimiento en el 2018. Lo anterior, se pudiera explicar por mejores niveles de infraestructura y transparencia de sus mercados (Herrington, 2017).

En relación al ecosistema financiero las diferencias en promedio de los países del nivel alto son más grandes, le siguen el nivel medio y, por último, el bajo. Esto pudiera ser causa de mayor diferencial en la parte del apoyo que reciben los nuevos emprendedores. El acceso a los mercados de capitales determina el crecimiento de las empresas, por lo que factores como cambios culturales de tolerancia al riesgo, menores niveles de riesgo y programas que fomenten el acceso a los mercados (Acemoglu et al., 2005) podrían disminuir las diferencias entre países y entre grupos. Asimismo, se explica por que existen diferencias en infraestructura financiera muy marcada entre los países emergentes y en desarrollo (Voelker, 2012), lo cual repercute en la gran distancia entre ecosistemas de alto y bajo nivel. Solo es destacable la labor en materia de políticas financieras realizadas en países como Chile Y China

En el ecosistema tecnológico, se presenta una situación parecida con el primer ecosistema ya que los países no cambian de grupo en el tiempo y esto es debido a las diferentes políticas en la parte tecnológica que se han aplicado. Mientras que países como China y Chile existen apoyos a sectores estratégicos, en la mayoría de los países latinoamericanos y en algunos asiáticos no se otorgan. En los países avanzados el emprendimiento productivo es cercano a la frontera tecnológica (Ács, et al., 2019, Lundström, et al., 2014). En cambio, economías latinoamericanas no se tienen fortalezas tecnológicas.

En segundo lugar, es importante determinar si el ecosistema se encuentra en equilibrio, entre los agentes que lo constituyen y el entorno físico que lo rodea, o está abierto al exterior, es decir, variables exógenas que lo afectan (McKenzie y Sud, 2009). En los ecosistemas financiero encontramos mayores distancias en el nivel alto de los emprendedores, mientras que en el ecosistema tecnológico las diferencias de este mismo 
nivel tienden a disminuir en el 2018. La mejora de los ecosistemas financieros y tecnológicos dependerá de la cooperación de los actores y de la resiliencia con la que enfrentan las empresas los cambios externos, en donde las transformaciones y transiciones son básicas (O'Connor et al., 2018). Por lo que pensamos que las economías emergentes deberán de invertir en empresas nuevas y del sector tecnológico y financiero que les ayude a cambiar de nivel; permitiendo apertura y transparencia en las regulaciones y normativas e incorporando programas que han sido exitosos en economías como la chilena para el caso de los países latinoamericanos o en su caso a la economía China para los países asiáticos.

Finalmente, las diferencias culturales manifestadas por el Foster et al. (2013) como la cultura de respeto al emprendimiento, cultura que promueva la tolerancia al riesgo y al fracaso y la confianza (Mihet, 2013) pudieran ser la casusa de las diferencias entre los grupos de los diferentes ecosistemas estudiados. Es recomendable que, tanto sector público como privado, instrumenten medidas orientadas a las empresas, que les permitan mayor adaptación ante los cambios, lo que ayudaría a los países a contar con ecosistemas de calidad. De esta forma facilitarían a los emprendedores realizar actividades emprendedoras y mejorar el bienestar económico no importando la región, el país o el sector al que pertenezca un nuevo emprendimiento.

Dada la naturaleza explicativa de esta investigación a nivel global en base a fuentes secundarias y a diferencias entre los países. Para futuras investigaciones, se tiene contemplado realizar un análisis de los ecosistemas del emprendedor a partir de sectores. Otra posible línea de investigación podría incluir un estudio con una mayor profundidad a través del diseño de un instrumento y aplicarse a nuevos emprendimientos con el propósito de analizar los impactos institucionales, culturales y ambientales y sus efectos en el bienestar de una región en particular.

\section{Referencias}

[1] Acemoglu D., Johnson S. y Robinson J. (2005). Institutions as a Fundamental Cause of Long-Run Growth. En Aghion, P. y Durlauf, S. (Ed.). Handbook of Economic Growth, 285-472, Amsterdam: Elsevier. https://doi.org/10.1016/s1574-0684(05)01006-3

[2] Acs Z. J., Autio E. y Szerb L. (2014). National Systems of Entrepreneurship: Measurement Issues and Policy Implications. Research Policy, 43(1). 476-494. https://doi.org/10.1016/j.respol.2013.08.016

[3] Ács Z. J., Szerb L., Lafuente E. y Gábor M. (2019). The Global Entrepreneurship Index. London: The Global Entrepreneurship and Development Institute

[4] Ahlstrom D. y Bruton G. D. (2008). Venture capital in emerging economies: Networks and institutional change. Entrepreneurship Theory and Practice, 30, 299-320. https://doi.org/10.1111/j.1540-6520.2006.00122.x

[5] Ahmad N. y Hoffmann A. (2008). A Framework for Addressing and Measuring Entrepreneurship. OECD Statistics. Recuperado de: http://ssrn.com/abstract=1090374

[6] Arroyo J. y Maté C. (2009). Forecasting histogram time series with k-nearest neighbours methods, International Journal of Forecasting, 25 (1), 192-207 https://doi.org/10.1016/j.ijforecast.2008.07.003

[7] Audretsch D. B. (2003). Standing on the Shoulders of Midgets: The U.S. Small Business Innovation Research Program. Small Business Economics, 20 (2), 129-136

[8] Autio E., Kenney M. F., Mustar P., Siegel D. S. y Wright M. T. (2014). Entrepreneurial innovation: The importance of context. Research Policy, 43(7), 1097-1108- https://doi.org/10.1016/j.respol.2014.01.015

[9] Autio E., Nambisan S., Thomas L. y Wright M. (2018). Digital affordances, spatial affordances, and the genesis of entrepreneurial ecosystems. Strategic Entrepreneurship Journal, 12(1), 72-95. https://doi.org/10.1002/sej.1266

[10] Avanzini, D. B. (2011). Designing composite entrepreneurship indicators. En Entrepreneurship and Economic Development. London: Palgrave Macmillan, 37-93. https ://doi.org/10.1057/9780230295155_3

[11] Banco Mundial. (2020). Global Economic Prospect. 6 de mayo de 2020, de OECD Sitio web: https://www. worldbank.org/en/publication/global-economic-prospect 
[12] Bruton G. D., Ahlstrom D., y Obloj K. (2008). Entrepreneurship in emerging economies: Where are we today and where should the research go in the future. Entrepreneurship Theory and Practice, 32(1), 1-14. https://doi.org/10.1111/j.1540-6520.2007.00213.x

[13] Cover T. M. y Hart P. E. (1967). Nearest neighbor pattern classification. IEEE Transactions on Information Theory, 13, 21-27 https://doi.org/10.1109/tit.1967.1053964

[14] Dasarathy B.V. (1991). Nearest neighbor (NN) norms: NN pattern classification techniques. Los Alamitos, CA: IEEE Computer Society Press

[15] Dattée, B., Alexy, O., \& Autio, E. (2018). Maneuvering in poor visibility: How firms play the ecosystem game when uncertainty is high. Academy of Management Journal, 61(2), 466-498. https://doi.org/10.5465/amj.2015.0869

[16] De Clercq D., Danis W. M. y Dakhli M. (2010). The moderating effect of institutional context on the relationship between associated activity and new business activity in emerging economies. International Business Review, 19(1), 85-101. https://doi.org/10.1016/j.ibusrev.2009.09.002

[17] Feld B. (2012). Startup Communities: Building an Entrepreneurial Ecosystem in Your City. New York: Wiley. https://doi.org/10.1002/9781119204459

[18] Fernández-Rodríguez F., Sosvilla-Rivero S. y Andrada-Félix, J. (2004). Nearest-neighbour predictions in foreign exchange markets. En Computational Intelligence in Economics and Finance, Heidelberg Berlin: Springer, 297325. https://doi.org/10.1007/978-3-662-06373-6_14

[19] Finkenstadt B. y Kuhbier P. (1995). Forecasting nonlinear economic time series: a simple test to accompany the nearest neighbor approach. Empirical Economics. 20(2), 243-263 https://doi.org/10.1007/bf01205437

[20] Foster G., Shimizu C., Ciesinski S., Davila A., Hassan S. Z., Jia, N. y Morris R. (2013). Entrepreneurial ecosystems around the globe and company growth dynamics (September 2013). In Retrieved from World Economic Forum: http://www3.weforum.org/docs/WEF_EntrepreneurialEcosystems_Report_2013.pdf

[21] Geitlinger, S. (2016). Entrepreneurship in South Africa: a comparative study between the Global Entrepreneurship Monitor, the Global Entrepreneurship Index and opinions of industry experts in the entrepreneurship field (Doctoral dissertation, University of Pretoria).

[22] Gurdur D., El-khoury J. y Törngren M. (2019). Digitalizing Swedish industry: What is next? Computers in Industry, 105, 153-163. https://doi.org/10.1016/j.compind.2018.12.011

[23] Harrington K. (2016). Is your entrepreneurial ecosystem scaling? An approach to inventorying and measuring a region's innovation momentum. Innovations: Technology, Governance, Globalization, 11(1-2), 126-142. https : //doi.org/10.1162/inov_a_00252

[24] Henrekson M. (2005). Entrepreneurship: a weak link in the welfare state? Industrial and Corporate Change, 14(3), 437-467. https://doi.org/10.1093/icc/dth060

[25] Herrintong M. (2017). Global Entrepreneurship Monitor 2017 Global Report. Babson Park: Global Entrepreneurship Research Association. Recuperado: https://www.c4e.org.cy/reports/2017/gem-2016-2017-globalreport-web-version-1486181226.pdf. https://doi.org/10.1093/acrefore/9780190224851.013.156

[26] Hofstede G. y Milosevic D. (2011). Dimensionalizing cultures: The Hofstede model in context. Online readings in psychology and culture, 2(1), 1-17 https://doi.org/10.9707/2307-0919.1014

[27] Isenberg D. J. (2010). How to start an entrepreneurial revolution. Harvard business review, 88(6), 40-50

[28] Jain A. K. y Dubes R.C. (1988). Algorithms for Clustering Data. Englewood, Cliffs: Prentice-Hall

[29] Lawson C. (1999). Towards a competence theory of the region. Cambridge Journal of Economics, 23(2), 151-166

[30] Lorenz E. (1991). Dimension of weather and climate attractors. Nature, 353 (6341), 241-244 https://doi.org/10.1038/353241a0

[31] Malecki, E. J. (2018). Entrepreneurship and entrepreneurial ecosystems. Geography Compass, 12(3), 1-21 
[32] McKenzie B.M. y Sud M. (2009). Prolegomena to a new ecological perspective in entrepreneurship. Academy of Entrepreneurship Journal, 15(1), 43-60

[33] Mihet R. (2013). Effects of culture on firm risk-taking: a cross-country and cross-industry analysis. Journal of Cultural Economics, 37(1), 109-151. https://doi.org/10.1007/s10824-012-9186-2

[34] Mongrut S. y Juárez N. (2018). Valoración de emprendimientos: una perspectiva estratégica y conductual. Revista Mexicana de Economía y Finanzas, 13(3), 419-439. https://doi.org/10.21919/remef.v13i3.314

[35] Nambisan, S., y Baron R.A. (2013). Entrepreneurship in innovation ecosystems: Entrepreneurs' self-regulatory processes and their implications for new venture success. Entrepreneurship theory and practice, 37(5), 1071-1097. https://doi.org/10.1111/j.1540-6520.2012.00519.x

[36] O'Connor A., Stam E., Sussan F., Audretsch D.B. (2018). Entrepreneurial Ecosystems: The Foundations of Place-based Renewal. En O'Connor A., Stam E., Sussan F., Audretsch D. (Eds). Entrepreneurial ecosystems: Place-based transformations and transitions, 1-21. New York: Springer https://doi.org/10.1007/ 978-3-319-63531-6_1

[37] Oladunjoye O., Oladele P. O. y Akeke N. I. (2011). Entrepreneurship Development: A Panacea for Unemployment Reduction in Nigeria. Journal of Emerging Trends in Economics and Management Sciences, 2(4), 251-256

[38] Porter M. E. (1998). Clusters and the new economics of competition. Harvard Business Review, 76, 77-90

[39] Pušnik K. y Tajnikar M. (2010). Heterogeneity and Competitiveness of Entrepreneurial Processes in the European Union with Special Attention on Croatia as Candidate Country. South East European Journal of Economics and Business, 5(1), 7-18. https://doi.org/10.2478/v10033-010-0001-8

[40] Raluca O. (2011). The Journey to Competitiveness: EU Speeding up on the Road Paved With Knowledge and Innovation. Annals of the University of Oradea, Economic Science Series, 20(1), 118-124

[41] Reynolds P. D., Bosma N. y Autio, E. (2005). Global Entrepreneurship Monitor: Data Collection Design and Implementation 1998-2003. Small Business Economics, 24(3), 205-231. https://doi.org/10.1007/s11187-005-19801

[42] Robb A. M. (2002). Entrepreneurial Performance by Women and Minorities: The Case of New Firms. Journal of Developmental Entrepreneurship, 7(4), 383

[43] Rosiello A., Avnimelech G. y Teubal M. (2011). Towards a systemic and evolutionary framework for venture capital policy. Journal of Evolutionary Economics, 21(1), 167-189. https://doi.org/10.1007/s00191-010-0189-x

[44] Rousseau P. L. y Sylla R. (2003). Financial systems, economic growth, and globalization. En Globalization in historical perspective (pp. 373-416). University of Chicago Press. https://doi.org/10.7208/chicago/9780226065991.003.0009

[45] Schumpeter, J.A. (1934). The Theory of Economic Development: An Inquiry into Profits, Capital, Credit, Interest, and the Business Cycle. Cambridge, MA: Harvard University Press

[46] Scott A. (1986). High technology industry and territorial development: the rise of the Orange county complex 1955-1984. Urban Geography, 7(1), 3-45. https://doi.org/10.2747/0272-3638.7.1.3

[47] Stam (2014) The Dutch entrepreneurial ecosystem. Recuperado: http://dx.doi.org/10.2139/ssrn.2473475

[48] Stam E. (2015). Entrepreneurial ecosystems and regional policy: a sympathetic critique. European Planning Studies, 23(9), 1759-1769. https://doi.org/10.1080/09654313.2015.1061484

[49] Stam E. (2018). Measuring entrepreneurial ecosystems. En A. O'Connor, E. Stam, F. Sussan,y D. Audretsch (Eds.), Entrepreneurial ecosystems: Place-based transformations and transitions, 173-197, New York: Springer. https://doi.org/10.1515/zfw-2019-0001

[50] Stone, C.J. (1977)- Consistent nonparametric regression. The Annals of Statistics, 5(4), 595-620

[51] Storper M. (1995). The resurgence of regional economies, ten years later: the region as a nexus of untrades interdependences. European Urban and Regional Studies, 2(3), 191-221. https://doi.org/10.1177/096977649500200301 
[52] Sugihara G. y May R.M. (1990). Nonlinear forecasting as a way of distinguishing chaos from measurement error in time series. Nature, 344 (6268), 734-741. https://doi.org/10.1038/344734a0

[53] Sur A., Chowdhury A., Chowdhury J. G., y Das S. (2013). Automatic Clustering Based on Cluster Nearest Neighbor Distance (CNND) Algorithm. En Proceedings of the International Conference on Frontiers of Intelligent Computing: Theory and Applications (FICTA) (pp. 189-196). Berlin, Heidelberg: Springer. https://doi.org/ 10.1007/978-3-642-35314-7_22

[54] Tabellini G. (2010). Culture and institutions: economic development in the regions of Europe. Journal of the European Economic association, 8(4), 677-716.. https://doi.org/10.1111/j.1542-4774.2010.tb00537.x

[55] Tallman S., Jenkins M., Henry N. y Pinch S. (2004). Knowledge, clusters, and competitive advantage. Academy of Management Review, 29(2) 258-271. https://doi.org/10.5465/amr.2004.12736089

[56] Treichel, M. y Scott J. A. (2006). Women-Owned businesses and access to bank credit: Evidence from three surveys since 1987. Venture Capital, 8(1), 51-67. https://doi.org/10.1080/13691060500453726

[57] Verheul I., Wennekers S., Audretsch D. y Thurik R. (2002). An eclectic theory of entrepreneurship: policies, institutions and culture. En Entrepreneurship: Determinants and policy in a European-US comparison (pp. 11-81). Boston, MA: Springer. https://doi.org/10.1007/0-306-47556-1_2

[58] Voelker T.A. (2012). Entrepreneurial ecosystems: Evolutionary paths or differentiated Systems? Business Studies Journal, (4)2, 43-61

[59] Wu, X., Zhang, C., \& Zhang, S. (2004). Efficient mining of both positive and negative association rules. ACM Transactions on Information Systems , 22(3), 381-405.. https://doi.org/10.1145/1010614.1010616

[60] Wansleben L. (2013). Dreaming with BRICs' Innovating the classificatory regimes of international finance. Journal of Cultural Economy, 6(4), 453-471. https://doi.org/10.1080/17530350.2012.756826

[61] Weber M. (1965). The protestant ethic and the spirit of capitalism. In 7th impression (first published in 1930). London: Unwin University Book

[62] Xu, Y., Zhu, Q., Fan, Z., Qiu, M., Chen, Y., Liu, H. (2013). Coarse to fine K nearest neighbor classifier. Pattern recognition letters, 34(9), 980-986. https://doi.org/10.1016/j.patrec.2013.01.028

[63] Yiu D.W., Lau C.M. y Bruton, G. D. (2007). International venturing by emerging economy firms: the effects of firm capabilities, home country networks, and corporate entrepreneurship. Journal of International Business Studies, 38(4), 519-540. https://doi.org/10.1057/palgrave.jibs.8400278

[64] Zhang, S., Li, X., Zong, M., Zhu, X., \& Wang, R. (2017). Efficient KNN classification with different numbers of nearest neighbors. IEEE transactions on neural networks and learning systems, 29(5), 1774-1785. https://doi.org/10.1109/tnnls.2017.2673241 